\title{
Mapping of MAC Address with Moving WiFi Scanner
}

\author{
Arief Hidayata ${ }^{\mathrm{a}, 1, *}$, Shintaro Terabe ${ }^{\mathrm{a}, 2}$, Hideki Yaginuma ${ }^{\mathrm{a}, 3}$ \\ ${ }^{a}$ Transportation Planning Laboratory, Department of Civil Engineering, Tokyo University of Science, \\ 2641 Yamazaki, Noda 278-8510, Japan \\ ${ }^{1}$ ariefhidayat06@hotmail.com*; ${ }^{2}$ terabe@rs.noda.tus.ac.jp; ${ }^{3}$ yaginuma@rs.tus.ac.jp \\ * corresponding author
}

ARTICLE INFO ABSTRACT

Article history:

Received : 09/15/2017

Revised : 10/04/2017

Accepted : 10/04/2017

Keywords:

WiFi Scanner

MAC Address

Processing, Bus
Recently, Wifi is one of the most useful technologies that can be used for detecting and counting MAC Address. This paper described using of WiFi scanner which carried out seven times circulated the bus. The method used WiFi and GPS are to counting MAC address as raw data from the pedestrian smartphone, bus passenger or WiFi devices nearfrom the bus as long as the bus going around the route. There are seven processes to make map WiFi data. The results of the process obtained a map that contains data MAC Address.

Copyright (C) 2017 International Journal of Artificial Intelegence Research.

All rights reserved.

\section{Introduction}

Recently, technological advances require for faster data retrieval. BIG DATA era requires prosessing both of these things technology input in it. In this case, counting of data MAC address using technology such as Bluetooth, smartphones or WiFi developed. Some researchs have applied Bluetooth and WiFi sensor to know the position of the vehicle and human. However, most of them used considerable cost and a fairly limited time with high maintenance levels. Some methods of count provided high cost and difficult to apply for some further research.

WiFi is one of the most widely used method today for smartphone, laptop, tablet and some other devices that are currently in high demand around the world and very close to human who do a movement. WiFi becomes one of the most useful options for getting movement or MAC address data. Every single networking device is equipped with a globally unique hardware address called MAC address
[1]-[3]. WiFi tracking provides a good approximation to crowd densities because WiFi has a longer detection range, and so a greater area is covered by each sensor [4] and WiFi is being detected increasingly every year from portable devices [5].

In principle method, multiple sensors are used to record the unique Bluetooth or wireless fidelity (WiFi) media access control (MAC) address for each wireless communication device [2], [4]. WiFi MAC address can be used to identify a mobile device and it can be used to determine the location of a mobile device when it is combined with received signal strength at multiple locations [6]. The use of android is also widely used to detect pedestrian movements. Most smartphones, which have WiFi functions, usually sends probe request frame to associate with a WiFi access point (depends on device). The last research such as using WiFi devices paired permanently in strategic locations [7], and the use of software that must be installed on a smartphone where most pedestrians do not install the software 
[8]. This research will describe and explain WiFi scanner data in Obuse Town, one of tourism place in Japan. This paper method used WiFI scanner as a counter or detector for MAC address data. This WiFi Scanner placed on the bus as moving detector. The Novelty of this research are moving detector (WiFi Scanner) data, using simple equipment and lack of used energy source.

\section{Method}

Location of the study in Obuse Town Kamatakai District, Nagano Prefecture in October 2016. Obuse is one of the top tourist destinations in Japan. [9].

\section{A. Field Experiment}

Obuse Town has a shuttle bus called Circle Bus "Romango". Romango bus is a Hop On-Hop Off bus. It has seven circulation journeys from bus stop number one to bus stop number nine. From morning 9:50 until 17:50.

The Obuse town operates two circular buses on every Saturday and Sunday. The circular route is approximately $15 \mathrm{~km}$ long and it takes 50 minutes for a round trip. Each bus starts in every 30 minutes from 9 am to 4 pm. There are two types Romango bus no. 1 and no. 2 on Figure 1.

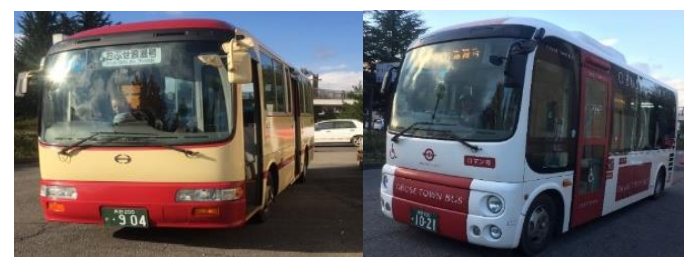

Fig. 1. "Romango Circle Bus" No.1 and No. 2

The overall route length is 8805 meters. The longest route is from bus stop no. 1 to bus stop no. 2 that is 2675.92 meters long. For the shortest route length that is bus stop number 4 to bus stop no.5 with length 304.59 meters.

\section{B. WiFi Equipment}

This study used WiFi Scanner to acquire data. This Scanner using minicomputer Raspberry Pi 2 B V1.1 [11] as a WiFi scanner, GPS tracking, and micro USB power source to save the data [12]. Mobile battery keep
WiFi Scanner stay on for 12 hours. The result of collecting and analyzing data, it is possible to grasp data, such as spatial flow and distribution of information device users. In this survey, we placed WiFi Scanner as Figure 2 in the bus. WiFi Scanner collected MAC Address were changed to point on the map.

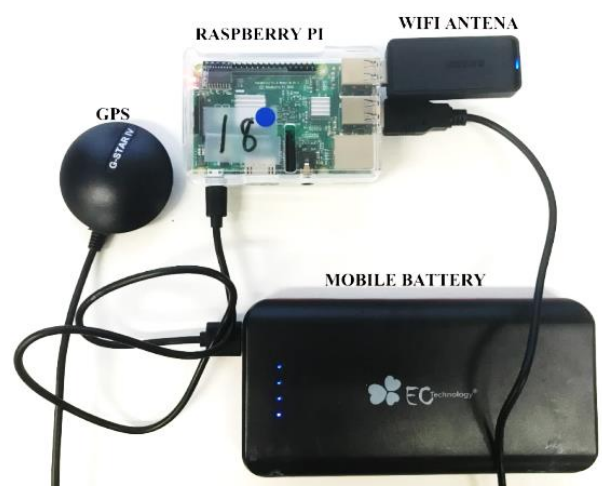

Fig. 2.WiFi Scanner, GPS and Mobile Battery

\section{Experiment}

WiFi scanner put on the board of a bus (Figure 3). A scanner device mounted on the shuttle bus from $09.50-17.50$. The installation of the tool is not complicated, put in a place that does not interfere with bus drivers and bus passengers. The WiFi tool is installed on buses no. 1 installed near the left window and no. 2 placed above the driver.

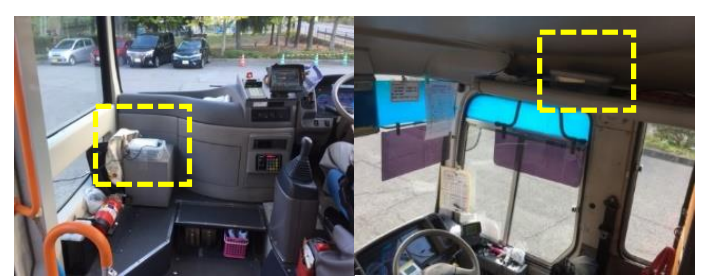

Fig. 3. Installing WiFi Scanner on Bus No.1 And Bus No.2

It can detect people who have those MAC address devices such as smartphones and tablets within 200-300 meters (accuracy) radius approximately (Figure 4). This scanner records unique identification code of detected mobile devices. 


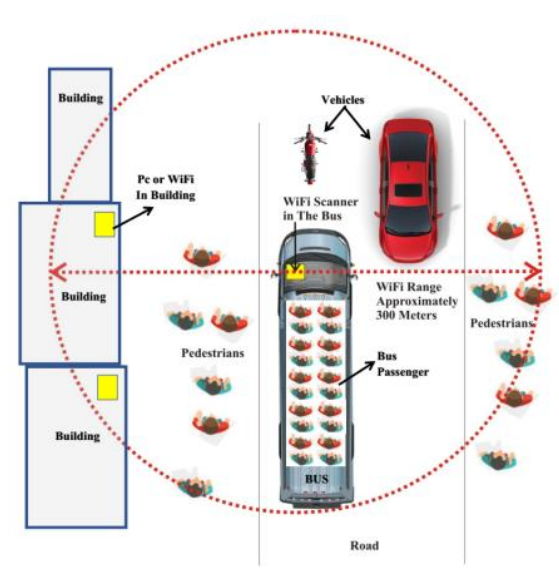

Fig. 4.WiFi Scanner Approximately Range

\section{Result : Structure and Mapping Data}

The result are GPS log and WiFi log as raw data. GPS log contain time, latitude and longitude data. WiFi log contains time and MAC ID (address). Structure data between WiFi and GPS is shown in Tabel 1.

Table 1. Structure of WiFi and GPS Data

\begin{tabular}{cl}
\hline & Day \\
\cline { 2 - 2 } GPS & Time \\
\cline { 2 - 2 } Data & Latitude \\
\cline { 2 - 2 } & Latitude compass direction \\
\cline { 2 - 2 } & Longitude \\
\cline { 2 - 2 } WiFi & Longitude compass direction \\
Data & day \\
\cline { 2 - 2 } Structure & Type \\
\cline { 2 - 2 } & Mac Address \\
\hline
\end{tabular}

MAC or Media Access Control is the unique ID or address assigned to each network device to be used as an identification code. (Figure 6). There are seven steps processing to mapping the MAC address data;

- The first step is combine raw data between WiFi log and GPS log to get WiFi data containing latitude and longitude. Keywords to combine data is "Time" data. The table data please refer to Tabel 2.
Table 2. WiFi and GPS Data Combine

\begin{tabular}{rlrr}
\hline Time & Mac & Latitude & Longitude \\
\hline 9:50:02 & 695f5323e896ab94951101fc410dcc1f & 3641.307 & 13817.68 \\
\hline 9:50:02 & 695f5323e896ab94951101fc410dcc1f & 3641.307 & 13817.68 \\
\hline 9:50:02 & 695f5323e896ab94951101fc410dcc1f & 3641.307 & 13817.68 \\
\hline 9:50:02 & 695f5323e896ab94951101fc410dcc1f & 3641.307 & 13817.68 \\
\hline 9:50:02 & af700e83c72c5453afc08284bbbae17f & 3641.307 & 13817.68 \\
\hline 9:50:02 & e65ba1616bbd6abd6db2e7cd06fecb53 & 3641.307 & 13817.68 \\
\hline 9:50:02 & e65ba1616bbd6abd6db2e7cd06fecb53 & 3641.307 & 13817.68 \\
\hline 9:50:07 & 3170fce3fe51cc10aaa1e927c6340c0c & 3641.307 & 13817.68 \\
\hline $\int$ & $\int$ & $\int$ & $\int$ \\
\hline 17:32:00 & 927c2d6fda58035a9ef66b847477eeb6 & 3641.935 & 13818.79 \\
\hline
\end{tabular}

- The second step is to eliminate error of data using loop and pivot data to make unique data at once to.

- The third is to convert coordinate system from latitude and longitude to universe transverse Mercator (UTM)

- The fourth is to import data to GIS.

- The fifth is to show point data from csv.

- The sixth is to make shapefile data from point.

- The seventh is to make map of MAC address.

All of processing using Anaconda 1.5 Jupiter Notebook 5.0, Python 2.7, Microsoft Excel 2010 and QGIS software. Number of MAC Address collected from 09:00 to 18:00 are 71630. It is illustrated along the bus route. The flowchart of processing data and mapping of MAC address are shown in Figure 6.

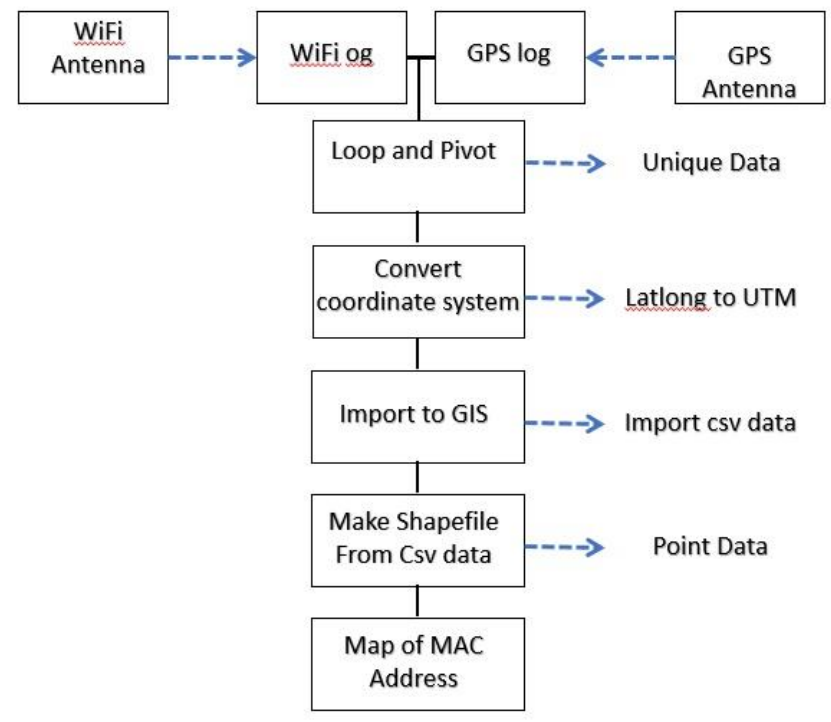

Fig. 5.Flowchart of Processing Mapping Data 
Based on the results of the processing with the distribution of MAC address in the map. It shown MAC Address of WiFi and GPS data are regularly located around the road in Obuse Town. The MAC Address data is the same as the bus line where the WiFi tool is located. This data becomes preliminary data that can be analyzed further to determine bus passengers or non-passenger. The map shown in Figure 6.

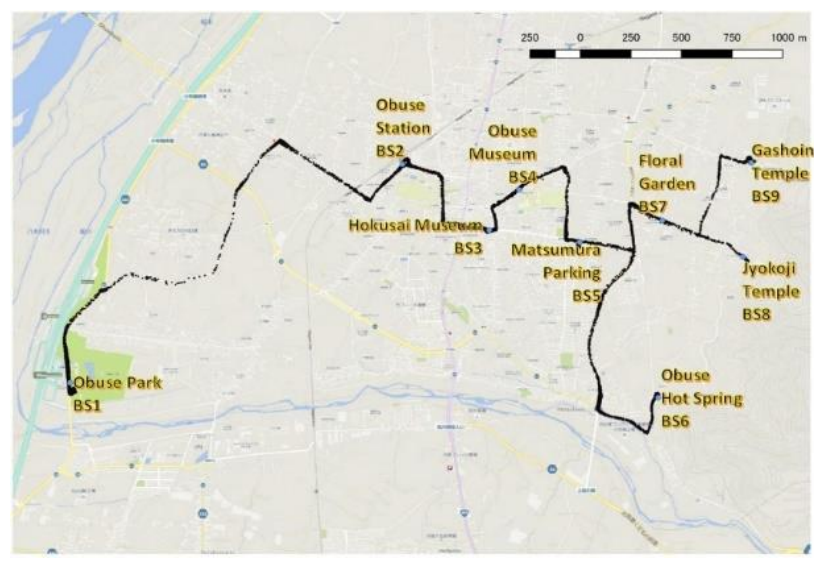

Fig. 6. Mapping of MAC Address

\section{Conclusion and Future Work}

The results of this paper obtained with the use of WiFi scanner on data collecting are:

- The MAC Address could be detected by location on map.

- Distance range of WiFi scanner as far as 200-300 meters is ideal distance for retrieving MAC address data.

- WiFi Scanner data retrieval is powerfull for long time data retrieval.

For Future work this data can be distinguished as passenger data (travel data) and non-passenger (pedestrian, vehicle and building) data according to this raw data.

\section{Acknowledgements}

The authors would like to thank all those who have contributed to this paper. Thanks to members of Transportation Planning Laboratory, Department of Civil Engineering, Tokyo University of Science (TUS) which has provided survey, data and much assistance in supporting for this paper.

\section{References}

[1] D. Al-Husainy and M. Fadhil, "MAC Address as a Key for Data Encryption," Int. J. Comput. Sci. Inf. Secur., pp. 83-87, 2013.

[2] J. Freudiger, "Short: How Talkative is your Mobile Device? An Experimental Study of Wi-Fi Probe Requests," WiSec '15 Proc. 8th ACM Conf. Secur. Priv. Wirel. Mob. Networks, pp. 1-6, 2015.

[3] M. Asija, "MAC Address," vol. 3, no. 1, pp. 41-60, 2016.

[4] M. Dunlap, Z. Li, K. Henrickson, and Y. Wang, "Estimation of Origin and Destination Information from Bluetooth and Wi-Fi Sensing for Transit," Transp. Res. Board 95th Annu. Meet., no. 2595, pp. 11-17, 2016

[5] R. Nishide and H. Takada, "Detecting Pedestrian Flows on a Mobile Ad Hoc Network and Issues with Trends and Feasible Applications," vol. 6, no. 1, pp. 108-117, 2013.

[6] Z. Xu et al., "Pedestrain Monitoring System using Wi-Fi Technology And RSSI Based Localization," Int. J. Wirel. Mob. Networks, vol. 5, no. 4, pp. 17-34, 2013.

[7] A. Lesani, "Development and Testing of a Real-Time WiFi-Bluetooth System for Pedestrian Network Monitoring and Data Extrapolation," no. January, pp. 1-13, 2016.

[8] N. Shlayan, A. Kurkcu, and K. Ozbay, "Exploring pedestrian bluetooth and WiFi detection at public transportation terminals," IEEE Conf. Intell. Transp. Syst. Proceedings, ITSC, pp. 229-234, 2016.

[9] Nagano Prefecture Goverment, "Obuse TouristGuide," 2016.

[10] Wikipedia, "Wikipedia," 2008. [Online]. Available: https://en.wikipedia.org/wiki/Obuse,_Naga no.

[11] Raspberry, "RASPBERRY PI 2 MODEL B," 2015. [Online]. Available: https://www.raspberrypi.org/products/rasp berry-pi-2-model-b/.

[12] Raspberry, "Raspberry Pi 2 Model B 1GB Technical Specifications," 2015. [Online]. 
Available:

https://www.element14.com/community/d
ocs/DOC-73827/1/raspberry-pi-2-model-b1gb-technical-specifications\#techspecs. 